Myiasis of Mandible Due to Lucilia sericata, in Diabetic Woman

\title{
Patient: A Case Report
}

\author{
Mona Roozbehani ${ }^{1}$, Jebreil Shamseddin ${ }^{2}$, Maryam Moradi ${ }^{1,{ }^{*}}$ and Leila Masoori ${ }^{1}$ \\ ${ }^{1}$ Department of Medical Parasitology, Faculty of Medicine, Iran University of Medical Sciences, Tehran, Iran \\ ${ }^{2}$ Infectious and Tropical Diseases Research Center, Hormozgan Health Institute, Hormozgan University of Medical Sciences, Bandar Abbas, Iran \\ "Corresponding author: Department of Medical Parasitology, Faculty of Medicine, Iran University of Medical Sciences, Tehran, Iran. Tel: +98-2182032103, Email: \\ dicentra2003@yahoo.com
}

Received 2017 August 08; Revised 2018 June 26; Accepted 2018 August 05.

\begin{abstract}
Introduction: Myiasis can be defined as the infestation of living tissues of humans and animals by dipterous eggs or larval stages that can penetrate the skin and soft tissues.

Case Presentation: The aim of the study was to report an uncommon case of insect infestation in human tissues, called myiasis. The patient was a 62-year-old woman, with the larval presence in the jaw, who suffered from diabetes and anemia. She was originally from the rural areas of Khuzestan province, the southwest of Iran. She had a wound on her mandible with a foul-smelling mouth before preparing for coronary artery bypass grafting (CABG) surgery. Her mandible and mouth were infested by 50 live larvae after three days of CABG. The larvae were examined in the pathology and parasitology department of the laboratory and definitely identified as Lucilia sericata.

Conclusions: Myiasis can be a risk for elderly people, especially old patients. The primary control method is the prevention and repulsion of adult flies before they can cause any damage.
\end{abstract}

Keywords: Myiasis, Lucilia sericata, Diabetic Patient

\section{Introduction}

Myiasis is considered as the infestation of living tissues of humans and animals by dipterous eggs or larvae. This situation is engendered by several fly species. Three main families of flies are involved in myiasis including Calliphoridae (tumbu flies, screwworms, green bottle, and blue bottle flies), Sarcophagidae (flesh flies), and Oestridae (warble flies and botflies). The larvae of the common house fly Musca domestica have also been identified, especially in neglected wounds $(1,2)$. The common green bottle fly, Lucilia sericata (Meigen), formerly Phaenicia sericata, is a common guest to carcasses, feces, and garbles. L. sericata has a substantial role in forensic science, medical, and veterinary affairs. In forensic science, the larvae or maggots help determine the period of insect colonization. The insect multiplication is related to the time of death and assists the detectives in their investigations. Medical treatment using maggot therapy can help researchers to ameliorate infectious wounds and infections that seem incurable. In the field of veterinary medicine, feeding by larval L. sericata can cause critical losses in animals and decrease their products (3).
Myiasis is classified into three types including obligatory myiasis in which fly species need the living tissue to lay eggs directly onto the intact skin, accidental myiasis in which insects lay eggs on food appliances and make infection when eaten, and semi-specific myiasis in which flies spread eggs directly on necrotic or trauma wounds (4). Larvae consume living or dead host tissues in a short period. They can typically cause disease or damage to different parts of the body that is categorized by the site of infection including cutaneous, nasopharyngeal, aural, ocular (ophthalmic myiasis), wound, intestinal, and urogenital myiasis (5).

\section{Case Presentation}

In September 2016, a 62-year-old Iranian woman was hospitalized in the CCU of Milad hospital in Tehran as a candidate for CABG (coronary artery bypass grafting). She was a diabetic patient with a background of anemia. The patient had a non-healing wound in her mandible with a foul-smelling mouth before preparing for CABG surgery. She was referred and admitted to the Milad hospital intensive care unit-open heart (ICU-OH) after CABG surgery. 
Her mandible and mouth were infested by 50 live larvae after three days of CABG surgery. The patient's mouth was washed with normal saline so that no more larvae were observed inside the mandible and mouth.

A physical examination showed no abnormality except for decreasing consciousness. Laboratory tests showed normal results, except for diabetes and anemia; therefore, insulin was prescribed for diabetes mellitus.

Larvae were examined in the pathology and parasitology Department of the hospital laboratory and the definite identification of Lucilia sericata was made based on the larval characteristics, such as smoothness of all larval stages, their taper shape, having a complete peritreme area surrounding their posterior spiracles that resembled eyes and located in the posterior part of the larvae and used for respiration (Figure 1). They stayed white through all three stages of formation, reaching a maximum of 12 - $18 \mathrm{~mm}$ before pupation (6), with the peritreme of the posterior spiracles being complete and stockade the button. As the larval emergence in the left mandible was detected after 10 days of hospitalization, nosocomial myiasis infection was ruled out. The symptoms of myiasis in our case were inconsequential and due to the patient's weakening consciousness, we did not see any complaint. The patient came from the rural areas of Mahshahr port in Khuzestan province, the southwest of Iran, in which the main occupation of inhabitants is keeping animals and farming with direct contact with myiasis-causing flies. Thus, the exact mechanism of infestation was not clear.

\section{Discussion}

Myiasis is the invasion of human or vertebrate tissues by a vast spectrum of fly larvae. It is common in the tropical and subtropical regions of Africa and America but occurs with remarkably lower frequencies in most other areas of the world. The clinical features of human myiasis are different according to the tissue or organ of infestation and the type and number of settled larvae. Flies causing obligatory myiasis make nasopharyngeal disturbances (nasal bots), digestive tract bots and even incriminate some or all the internal organs of animals and humans (7). Nosocomial myiasis takes place in hospitalized patients in which, open wounds or traumatic lesions of the disabled patient may be disturbed if flies exist in the environment and they are attracted to the venerable organs (8). Most known families of flies including Calliphoridae (blow flies), Sarcophagidae (flesh flies), Oestridae (bot flies), and Muscoidea (dung flies) have been identified as the main causes of specific or facultative myiasis in humans (9). L. sericata (Meigen), known as "common green bottle fly", is a member of the family Calliphoridae and one of the most important and known species in the genus. This fly is a common visitor to the carcass or rancid body, feces, and rubbish. L. sericata is ubiquitous, found all over the world, and harassing humans in America, Africa, and Asia (10). In veterinary medicine, the larval feeding habit of $L$. sericata can cause serious damages and even death in animals. In humans, however, infestation most often is restricted to subcutaneous tissues and causes furunculous injuries. It sometimes occurs in lesions and certain body cavities. Infestation and feeding activities of larvae at the cutaneous surface can rapidly result in the development of skin lesions, further egg laying, debilitating effects, and even death (11). Moreover, both larval and adult stages of L. sericata and Calliphoravicina (Diptera: Calliphoridae) can be the passive vectors of Mycobacterium avium subsp. avium, Mycobacterium avium paratuberculosis, and Mycobacterium avium hominissuis (12).

Oral (and adjacent oral soft tissue) myiasis is considered a type of ulcerative myiasis strongly related to poor oral and dental hygiene. Many other variables and agents can affect the incidence of the disease, including alcoholism, senescence, purulent or exudative lesions, gingival diseases, trauma, paralysis or lifelessness, and mental debility (13).

In our study, the patient was a diabetic, anemic woman with a poor socioeconomic background, who was living in rural regions. She had poor oral hygiene due to poverty and lack of facilities and sufficient public health education. It was supposed that the eggs were laid in the dirty wound directly by the flies. On the other hand, the warm humid climate, direct contact with domestic animals, poor hygienic conditions, and non-healing wound with halitosis attracted the flies to lay eggs on the wound.

To effectively prevent the fly invasion inside human houses, good sanitation and enhancement of environmental structures are very important. Environmental and hospital sanitation (e.g., installation of electrocuting devices to repel insects and flies) is also helpful. Some drugs can be administered orally or topically for the treatment of various types of myiasis including ivermectin in elderly patients in hospital wards.

Generally, the disease manifestations in human occur in undernourished individuals with poor hygiene or mentally disabled or retarded individuals who are incapable of protecting themselves against the attacks of flies. It also commonly occurs in some medical conditions, e.g. diabetes mellitus, psychiatric illness, and leprosy (14).

Furthermore, the treatment consists of the removal of larvae with forceps and daily washing and debridement of the wound until emerging new tissues. 

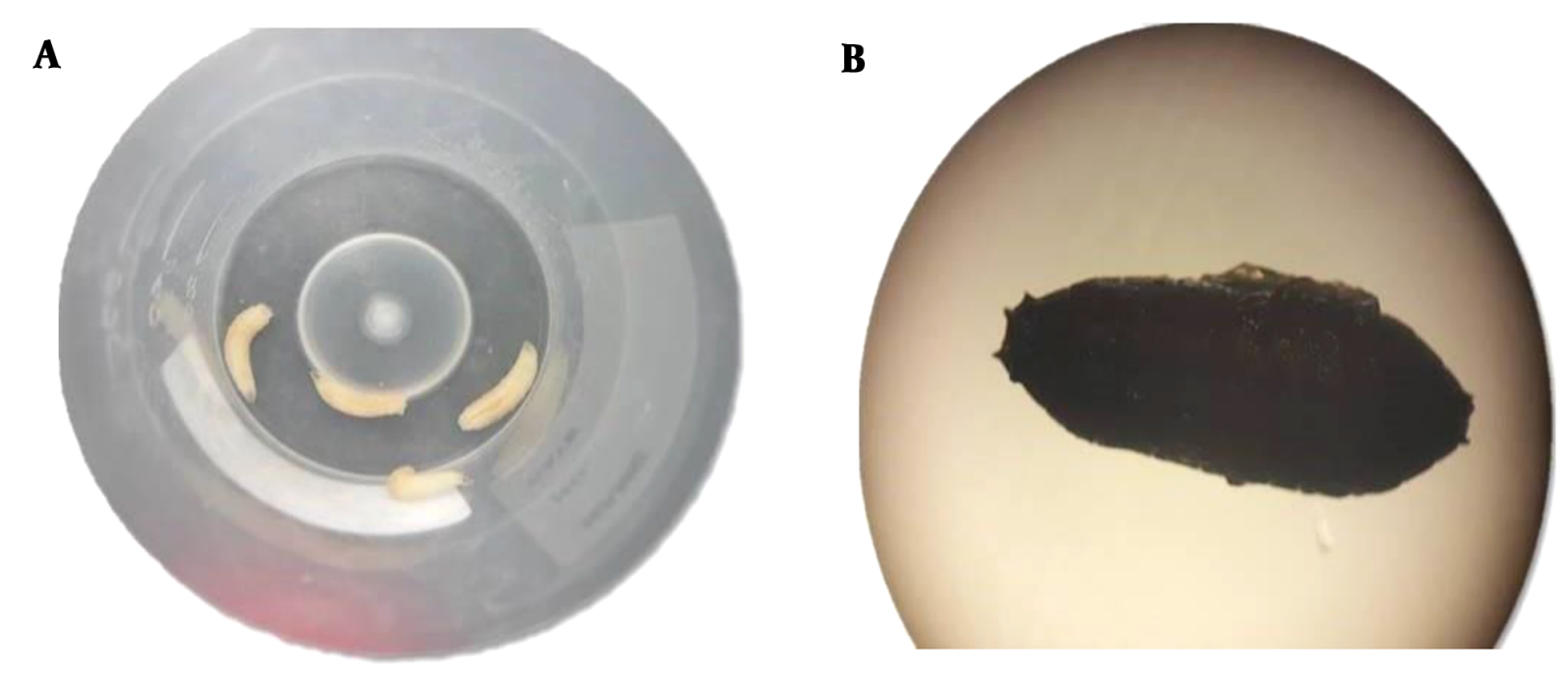

Figure 1. The larvae of Lucilia sericata (A), the pupae of Lucilia sericata (B) diagnosed for diabetic patient

\subsection{Conclusions}

Actually, myiasis can be a risk for elderly people, especially old patients. Proper hygiene, covering the open wounds, and immediate treatment are appropriate measures against flies that facultatively or obligatorily invade wounds, gangrenous tissues, or body holes and apertures. All tissues and organs can be the site of myiasis. Although this infestation sometimes can create severe problems, recently myiasis has been used to clean the wound to decrease the chance of secondary infections in some situations including diabetic foot and necrotic tissues to promote healing.

\section{Acknowledgments}

The authors would like to appreciate Prof. Dr. Ahmadreza Meamar for identifying Lucilia sericata larvae. We also thank the staff and head managers of Milad Hospital ICU-OH and medical laboratory that provided us with technical support for the development and conduct of this study.

\section{Footnotes}

Conflict of Interest: The authors declare that there are no conflict of interests.

Ethical Approval: All the procedures were confirmed by Milad Hospital Research Committee.

Funding/Support: The current study is a case report that has not used any financial resources or funds.

Patient Consent: Informed consent was obtained from the patient under study by the authors.

\section{References}

1. Saldarriaga W, Herrera E, Castro D. Myasis in uterine prolapse, successful treatment. Am J Obstet Gynecol. 2011;205(3):e5-6. doi: 10.1016/j.ajog.2011.03.019. [PubMed: 21530939].

2. Talari SA, Sadr F, Doroodgar A, alari MR, Gharabagh AS. Wound myiasis caused by Lucilia sericata. Arch Iran med. 2004;7(2):128-9.

3. Rueda LC, Ortega LG, Segura NA, Acero VM, Bello F. Lucilia sericata strain from Colombia: Experimental colonization, life tables and evaluation of two artificial diets of the blowfly Lucilia sericata (Meigen) (Diptera: Calliphoridae), Bogota, Colombia strain. Biol Res. 2010;43(2):197-203. doi: 10.4067/S0716-97602010000200008. [PubMed: 21031265].

4. Ramana KV. Human myiasis.JMed Microbiol Diagn. 2012;1(2). e105. doi: 10.4172/2161-0703.1000e105.

5. Ahmad NW, Ismail A, Jeffery J, Ibrahim S, Hadi AA, Ibrahim MN, et al. Aural myiasis in a neonate in peninsular Malaysia. Parasit Vectors. 2009;2(1):63. doi: 10.1186/1756-3305-2-63. [PubMed: 20003466]. [PubMed Central: PMC2797769].

6. Salimi M, Goodarzi D, Karimfar M, Edalat H. Human urogenital myiasis caused by Lucilia sericata (diptera: calliphoridae) and Wohlfahrtia magnifica (diptera: Sarcophagidae) in Markazi province of Iran. Iran J Arthropod Borne Dis. 2010;4(1):72-6. [PubMed: 22808392]. [PubMed Central: PMC3385545].

7. Kandi V, Lal SK, Akhila , Shruthi, Sandhya K, Simar H, et al. Persistent pediatric gastro-intestinal myiasis: A case report of fly larval infestation with Musca domestica with review of literature.J Glob Infect Dis. 2013;5(3):114-7. doi: 10.4103/0974-777X.116874. [PubMed: 24049366]. [PubMed Central: PMC3766333].

8. Hira PR, Assad RM, Okasha G, Al-Ali FM, Iqbal J, Mutawali KE, et al. Myiasis in Kuwait: Nosocomial infections caused by lucilia sericata and Megaselia scalaris. Am J Trop Med Hyg. 2004;70(4):386-9. doi: 10.4269/ajtmh.2004.70.386. [PubMed: 15100451].

9. Stevens JR, Wallman JF, Otranto D, Wall R, Pape T. The evolution of myiasis in humans and other animals in the old and new worlds (part II): Biological and life-history studies. Trends Parasitol. 2006;22(4):181-8. doi: 10.1016/j.pt.2006.02.010. [PubMed: 16507352]. 
10. Whitworth T. Keys to the genera and species of blow flies (diptera: Calliphoridae) of America North of Mexico. Proc Entomol Soc Wash. 2006

11. Broughan JM, Wall R. Control of sheep blowfly strike using fly-traps. Vet Parasitol. 2006;135(1):57-63. doi: 10.1016/j.vetpar.2005.08.005. [PubMed: 16188387].

12. Fischer OA, Matlova L, Dvorska L, Svastova P, Bartl J, Weston RT, et al. Blowflies Calliphora vicina and Lucilia sericata as passive vectors of Mycobacterium avium subsp. avium, M. a. paratuberculosis and M. a. hominissuis. Med Vet Entomol. 2004;18(2):116-22. doi: 10.1111/j.0269283X.2004.00477.x. [PubMed: 15189236].

13. Sharma A. Oral myiasis is a potential risk in patients with special health care needs. J Glob Infect Dis. 2012;4(1):60-1. doi: 10.4103/0974777X.93763. [PubMed: 22529629]. [PubMed Central: PMC3326961].

14. Shaunik A. Pelvic organ myiasis. Obstet Gynecol. 2006;107(2 Pt 2):501-3. doi: 10.1097/01.AOG.0000171116.38946.9e. [PubMed: 16449164]. 